\title{
Glasgow City Region sets a new benchmark for dealing with climate change
}

\author{
Kit England and Ruth Wolstenholme from environmental charity Sniffer and Graham Edmond of \\ Transport Scotland and ICE Scotland describe Glasgow City Region's pioneering risk assessment \\ for adapting to climate change. It offers numerous opportunities for civil engineers.
}

Climate Ready Clyde is an initiative of 13 organisations to develop an adaptation strategy and action plan for dealing with climate change in the Glasgow City Region. It includes local authorities, infrastructure operators, universities, the Scottish Environment Protection Agency and one of the region's two health boards, which together fund a technical secretariat, currently Sniffer, to lead the work.

The initiative has recently published a climate risk and opportunity assessment. As Scotland's flagship place-based adaptation initiative, it aims to raise the standard of risk assessment to inform adaptation planning and sets a benchmark for others to follow.

While other cities and regions have undertaken similar risk assessments, Glasgow City Region is the first place in the UK to have a publicly available prioritised set of risks and opportunities.

\section{Risk assessment approach}

The approach was based on the UK Climate Change Risk Assessment approach but modified to fit the local context. Relevant national risks for Glasgow City Region were selected, along with additional evidence and findings from new research.

For each risk or opportunity, its magnitude, adaptation shortfall and benefits to further action were assessed to assign one of four urgency scores. Over 100 stakeholders from across the city participated in validation and scoring workshops, as well as contributing through detailed discussion of the thematic assessments.

In parallel, Climate Ready Clyde commissioned an economic appraisal which highlighted climate change could cost Glasgow City Region $\mathrm{f} 400$ million a year by the 2050s, with higher damages from one-off extreme weather events. This highlights the vital need to adapt and start the journey now.

The approach of a robust risk assessment and economic appraisal offers various advantages. Using urgency scoring helps partners move beyond the technical details of risk assessment and scoring, providing

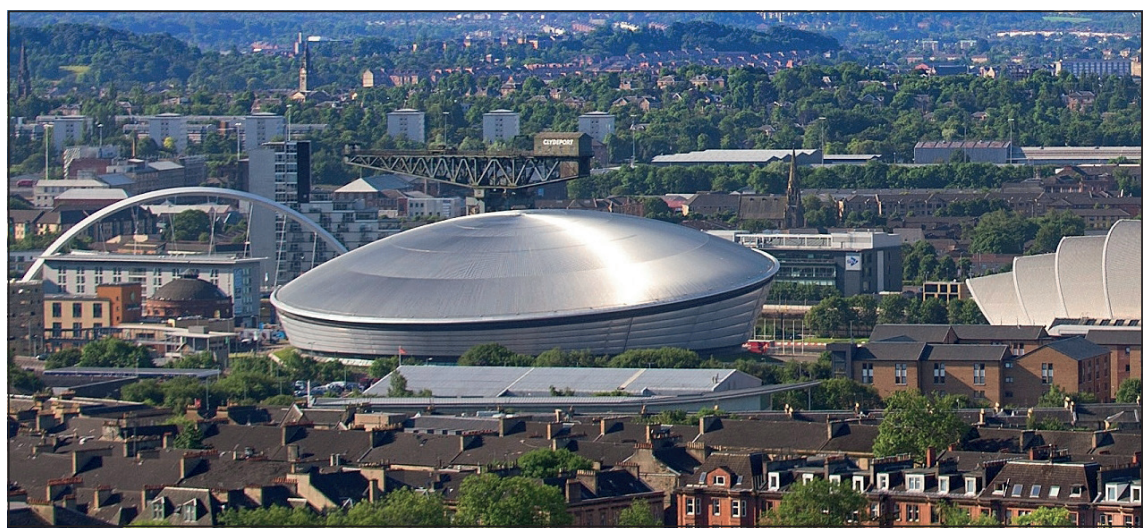

Properly future-proofing urban areas such as Glasgow City Region against climate change presents numerous opportunities for civil engineers (University of Glasgow)

strategic direction on the degree of action required in the next 5 years. Also, the assessment highlights that inaction also incurs a cost, laying the groundwork for a more nuanced assessment of costs and benefits.

The assessment also contains a number of innovative features. These include aligning with ISO 14091, the forthcoming standard on climate change risk assessment; assessing adaptive capacity and the current extent of climate justice considerations; and supporting Scottish policy outcomes and the UN sustainable development goals.

\section{Role of civil engineers}

The risk assessment highlighted the real benefits of adaptation in all aspects of the infrastructure life cycle. Infrastructure risks featured prominently as the long lifetime of infrastructure potentially locks these risks for the future. For example, exposures of bridges, railways and roads to damage from storms, coastal erosion, flooding and landslides were all highlighted as a priority.

The range of skills of civil engineers in designing, constructing and maintaining the vital infrastructure that supports society's continual well-being and vitality can and must be applied to finding ways to ensure existing infrastructure continues to function, and that new investments are screened for climate resilience. At the very least this is a growing source of further work and is central to the ethos of civil engineers that are creating a world for future generations.

Taken together, the assessment makes a compelling case for the civil engineering profession to create a step change in adaptation planning for future economic prosperity of the Glasgow City Region and indeed the wider world. 